\title{
Lactobacillus xiangfangensis sp. nov., isolated from Chinese pickle
}

\author{
Chun Tao Gu, ${ }^{1 \dagger}$ Fang Wang, ${ }^{2,3 \dagger}$ Chun Yan Li, ${ }^{1 \dagger}$ Fei Liu $^{1}$ \\ and Gui Cheng Huo ${ }^{1}$ \\ ${ }^{1}$ Key Laboratory of Dairy Science, Ministry of Education, Northeast Agricultural University, \\ Harbin 150030, PR China \\ ${ }^{2}$ State Key Laboratory of Agrobiotechnology, College of Biological Sciences, \\ China Agricultural University, Beijing 100193, PR China \\ ${ }^{3}$ College of Forestry, Southwest Forestry University, Kunming 650224, PR China
}

Correspondence

Chun Tao Gu

ctgu1977@yahoo.com.cn
The genus Lactobacillus belongs to the phylum Firmicutes, class Bacilli, order Lactobacillales, family Lactobacillaceae (Garrity et al., 2004). Lactobacilli are usually found in dairy products, fermented meat, sour doughs, vegetables, fruits, beverages, sewage, plant material, and in respiratory, GI and genital tracts of humans and animals (Felis \& Dellaglio, 2007).

In the present study, a Gram-positive bacterial strain, 3.1.1 ${ }^{\mathrm{T}}$, was isolated from traditional pickle in Heilongiiang Province, China. The bacterium was characterized by a polyphasic approach. The strains used in this study are listed in Table 1 . All strains were incubated aerobically at $30{ }^{\circ} \mathrm{C}$ on MRS medium (De Man et al., 1960).

Amplification of the 16S rRNA gene was performed using the primers of An et al. (2006). The pheS and rpoA genes were amplified using the primers of De Bruyne et al. (2007) and the protocols of Naser et al. (2005). The dnaK gene was amplified using the primers and protocol of Huang et al. (2010). Purification and sequencing of PCR products were carried out by the Shenggong Company in Shanghai, China. The resulting sequences, together with those of related strains obtained from the GenBank database were aligned by using CLUSTAL w. A phylogenetic tree was reconstructed

†These authors contributed equally to this work.

The GenBank/EMBL/DDBJ accession numbers for the 16S rRNA, pheS, rpoA and dnaK gene sequences of strain $3.1 .1^{\top}$ are HM443954, HM443974, HM443977 and HM443963, respectively. The accession number for the rpoA gene sequence of Lactobacillus fabifermentans LMG $24284^{\top}$ is HM443978.

A supplementary figure is available with the online version of this paper. using the neighbour-joining method with the maximum composite likelihood model. Bootstrap analysis was performed based on 1000 replicates. The MEGA4 package (Tamura et al., 2007) was used for all analyses. 16S rRNA gene sequence analysis of strain $3.1 .1^{\mathrm{T}}$ indicated that it belonged to the Lactobacillus plantarum species group, showing sequence similarities of $98.8,98.7,98.9,98.8$ and $98.5 \%$ to the type strains of L. plantarum subsp. plantarum, L. plantarum subsp. argentoratensis, Lactobacillus pentosus, Lactobacillus paraplantarum and Lactobacillus fabifermentans, respectively (Fig. 1). A large phylogenetic tree based on $16 \mathrm{~S}$ rRNA gene sequences showing the relationship between strain $3.1 .1^{\mathrm{T}}$ and the type strains of all defined species within the genus Lactobacillus is given in Supplementary Fig. S1 (available in IJSEM Online). Strain 3.1.1 ${ }^{\mathrm{T}}$ showed 75.1-84 \% pheS gene sequence similarities (Fig. 2), 92.8-93.6\% rpoA gene sequence similarities (Fig. 3) and $83.9-87.9 \%$ dnaK gene sequence similarities (Fig. 4) to the type strains of all species in the L. plantarum species group, respectively. pheS, $r p o A$ and dnaK gene sequence analyses (Figs 2-4) clearly show a higher resolution than the $16 \mathrm{~S}$ rRNA gene sequence

Table 1. Bacterial strains used in this study

\begin{tabular}{|ll|}
\hline Strain & \multicolumn{1}{c|}{ Isolation source } \\
\hline L. xiangfangensis sp. nov. $3.1 .1^{\mathrm{T}}$ & Pickle, China \\
L. fabifermentans LMG $24284^{\mathrm{T}}$ & Cocoa bean fermentation, Ghana \\
L. paraplantarum LMG $16673^{\mathrm{T}}$ & Beer, France \\
L. pentosus LMG $10755^{\mathrm{T}}$ & Sawdust fermentation \\
L. plantarum LMG $6907^{\mathrm{T}}$ & Pickled cabbage \\
\hline
\end{tabular}




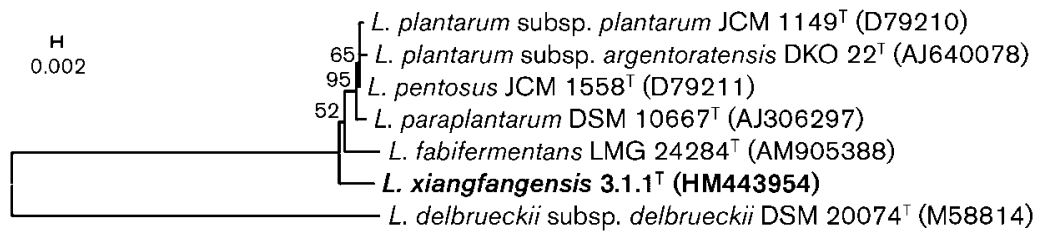

Fig. 1. Neighbour-joining tree showing the phylogenetic relationships of strain $3.1 .1^{\top}$ and related reference strains based on 16S rRNA gene sequences. Numbers at nodes are bootstrap values based on 1000 replicates. Bar, $0.2 \%$ substitutions per site.

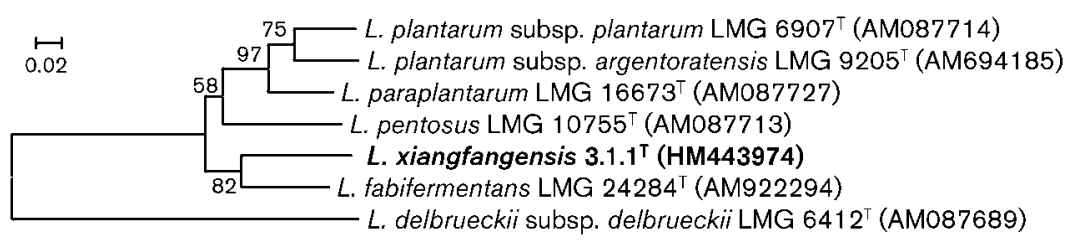

Fig. 2. Neighbour-joining tree showing the phylogenetic relationships of strain $3.1 .1^{\top}$ and related reference strains based on pheS gene sequences. Numbers at nodes are bootstrap values based on 1000 replicates. Bar, $2 \%$ substitutions per site.

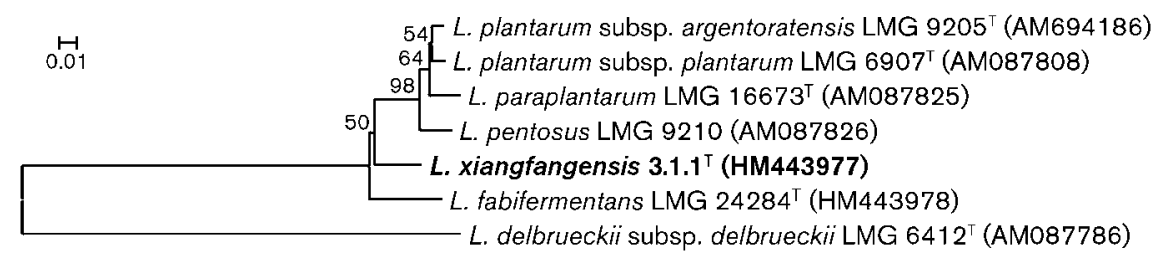

Fig. 3. Neighbour-joining tree showing the phylogenetic relationships of strain $3 \cdot 1 \cdot 1^{\top}$ and related reference strains based on rpoA gene sequences. Numbers at nodes are bootstrap values based on 1000 replicates. Bar, $1 \%$ substitutions per site.

analysis and suggest that strain $3.1 .1^{\mathrm{T}}$ represents a novel species within the genus Lactobacillus.

The API $50 \mathrm{CH}$ system (bioMérieux) was used to determine the carbohydrate fermentation profile of strain $3.1 .1^{\mathrm{T}}$. Test preparations were incubated at $30{ }^{\circ} \mathrm{C}$, and readings were made after $48 \mathrm{~h}$. The results are given in the species description below. Characteristics that differentiate strain $3.1 .1^{\mathrm{T}}$ from its closest relatives are summarized in Table 2.

Whole-cell fatty acids were analysed as fatty acid methyl esters with the MIDI Microbial Identification system. Cultures were incubated for 2 days at $30{ }^{\circ} \mathrm{C}$ on MRS solid medium. Fatty acid methyl esters were extracted and prepared according to the protocol of Sasser (1990).
Comparative fatty acid compositions of strain $3.1 .1^{\mathrm{T}}$ and phylogenetically related reference strains are given in Table 3.

DNA was prepared using Power-Microbial Maxi DNA isolation kits (Mo Bio Laboratories). DNA G $+\mathrm{C}$ content was measured using the thermal melting protocol of De Ley (1970) with Escherichia coli K-12 as the standard. DNA-DNA hybridizations were performed by the initial renaturation rate method (De Ley et al., 1970). The DNA G + C content of strain $3.1 .1^{\mathrm{T}}$ was $46.6 \mathrm{~mol} \%$. DNA-DNA hybridization values between strain $3.1 .1^{\mathrm{T}}$ and L. plantarum LMG $6907^{\mathrm{T}}$, L. pentosus LMG $10755^{\mathrm{T}}$, L. paraplantarum LMG $16673^{\mathrm{T}}$ and L. fabifermentans LMG $24284^{\mathrm{T}}$ were $27,26,41$ and $42 \%$, respectively, confirming that strain $3.1 .1^{\mathrm{T}}$ represents a novel species within the genus Lactobacillus.

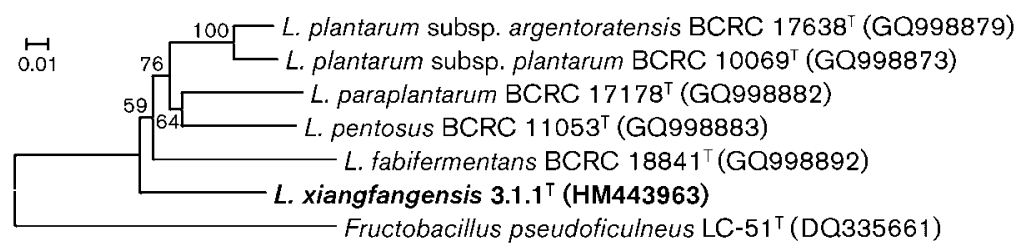

Fig. 4. Neighbour-joining tree showing the phylogenetic relationships of strain $3.1 .1^{\top}$ and related reference strains based on dnaK gene sequences. Numbers at nodes are bootstrap values based on 1000 replicates. Bar, 1\% substitutions per site. 
Table 2. Distinctive features of the carbohydrate fermentation profiles of strain $3.1 .1^{\top}$ and phylogenetically related species determined using API $50 \mathrm{CH}$

Strains: 1, L. xiangfangensis sp. nov. $3 \cdot 1.1^{\mathrm{T}}$ (data from this study); 2, L. fabifermentans LMG $24284^{\mathrm{T}}$ (De Bruyne et al., 2009); 3, L. paraplantarum (Curk et al., 1996); 4, L. pentosus (Zanoni et al., 1987); 5, L. plantarum subsp. plantarum (Bringel et al., 2005; Zanoni et al., 1987); 6, L. plantarum subsp. argentoratensis (Bringel et al., 2005). +, $90 \%$ or more strains positive;,$- 90 \%$ or more strains negative; $\mathrm{d}, 11-$ $89 \%$ of strains positive; D, delayed reaction (positive after incubating at $30{ }^{\circ} \mathrm{C}$ for 4 days).

\begin{tabular}{|c|c|c|c|c|c|c|}
\hline Characteristic & 1 & 2 & $\begin{array}{c}3 \\
(n=4)^{*}\end{array}$ & $\begin{array}{c}4 \\
(n=3)\end{array}$ & $\begin{array}{c}5 \\
(n=15)\end{array}$ & $\begin{array}{c}6 \\
(n=14)\end{array}$ \\
\hline Glycerol & - & - & - & + & $\mathrm{d}$ & d \\
\hline L-Arabinose & - & + & $\mathrm{d}$ & + & d & d \\
\hline D-Xylose & + & + & - & + & - & - \\
\hline Adonitol & + & - & - & - & - & - \\
\hline Rhamnose & - & - & $\mathrm{d}$ & $\mathrm{d}$ & d & d \\
\hline Dulcitol & - & - & - & d & - & d \\
\hline Sorbitol & + & - & $\mathrm{d}$ & + & d & d \\
\hline $\begin{array}{l}\text { Methyl } \alpha \text {-D- } \\
\text { mannopyranoside }\end{array}$ & - & - & - & - & d & - \\
\hline $\begin{array}{l}\text { Methyl } \alpha \text {-D- } \\
\text { glucopyranoside }\end{array}$ & - & - & - & $\mathrm{d}$ & d & d \\
\hline Amygdalin & - & + & + & + & + & + \\
\hline Arbutin & - & + & + & + & + & + \\
\hline Lactose & - & - & + & + & + & + \\
\hline Melibiose & - & - & + & + & + & + \\
\hline Melezitose & - & - & + & $\mathrm{d}$ & $\mathrm{d}$ & - \\
\hline Raffinose & - & - & $\mathrm{d}$ & + & $\mathrm{d}$ & d \\
\hline Starch & - & - & - & - & d & d \\
\hline Glycogen & - & - & - & - & $\mathrm{d}$ & d \\
\hline Turanose & - & - & $\mathrm{d}$ & d & d & d \\
\hline D-Arabitol & - & - & $\mathrm{d}$ & - & $\mathrm{d}$ & d \\
\hline Gluconate & - & - & + & - & d & d \\
\hline Aesculin & D & + & + & + & + & + \\
\hline
\end{tabular}

${ }^{\star} n$, Number of strains tested.

On the basis of the results presented here, strain $3.1 .1^{\mathrm{T}}$ is considered to represent a novel species of the genus Lactobacillus, for which the name Lactobacillus xiangfangensis sp. nov. is proposed.

\section{Description of Lactobacillus xiangfangensis sp. nov.}

Lactobacillus xiangfangensis (xi.ang.fang.en'sis. N.L. masc. adj. xiangfangensis pertaining to xiangfang district, located in Harbin, Heilongjiang Province, where the type strain was isolated).

Gram-positive. Non-spore-forming rods. Catalase is not produced. Facultatively anaerobic. Optimum growth temperature is $30{ }^{\circ} \mathrm{C}$; can grow at $37^{\circ} \mathrm{C}$. Acid is produced from ribose, $\mathrm{D}$-xylose, adonitol, galactose, glucose, fructose, mannose, mannitol, sorbitol, $N$-acetylglucosamine, salicin, cellobiose, maltose, sucrose, trehalose and gentiobiose. Acid
Table 3. Comparative fatty acid contents (\%) of strain $3.1 .1^{\top}$ and phylogenetically related reference strains

Strains: 1 , L. xiangfangensis sp. nov. $3.1 .1^{\mathrm{T}} ; 2$, L. fabifermentans LMG $24284^{\mathrm{T}}$; 3, L. paraplantarum LMG $16673^{\mathrm{T}}$; 4, L. pentosus $\mathrm{LMG} 10755^{\mathrm{T}}$; 5, L. plantarum LMG $6907^{\mathrm{T}}$. -, Not detected.

\begin{tabular}{|c|c|c|c|c|c|}
\hline Fatty acid & 1 & 2 & 3 & 4 & 5 \\
\hline $10: 0$ & - & - & - & 0.4 & - \\
\hline $12: 0$ & - & - & - & 0.3 & - \\
\hline $14: 0$ & - & 2.3 & - & 2.5 & - \\
\hline $16: 0$ & - & 38.4 & - & 30.4 & - \\
\hline $16: 1 \omega 5 c$ & - & 0.3 & - & - & - \\
\hline $17: 0$ & - & 0.2 & - & 0.1 & - \\
\hline $17: 0$ iso $3-\mathrm{OH}$ & 29.2 & - & - & - & - \\
\hline $17: 02-\mathrm{OH}$ & 27.6 & - & - & - & - \\
\hline $18: 0$ & - & 5.6 & 6.0 & 2.3 & - \\
\hline $18: 02-\mathrm{OH}$ & - & - & - & - & 7.7 \\
\hline 18:0 10-methyl, TBSA & - & - & - & - & 43.9 \\
\hline $18: 12-\mathrm{OH}$ & 4.4 & - & - & - & - \\
\hline $18: 1 \omega 5 c$ & - & - & - & - & 6.1 \\
\hline $18: 1 \omega 9 c$ & - & 20.1 & 22.1 & 12.0 & - \\
\hline $19: 0$ iso & - & - & - & 3.8 & - \\
\hline $19: 1$ iso $\mathrm{I}$ & - & 1.1 & 1.3 & - & 42.3 \\
\hline $16: 1 \omega 6 c$ and/or $16: 1 \omega 7 c$ & - & 2.5 & 2.4 & 4.4 & - \\
\hline $18: 2 \omega 6,9 c$ and/or $18: 0$ anteiso & - & 0.7 & 0.8 & 0.6 & - \\
\hline 19: $1 \omega 6 c$ and/or $19: 0$ cyclo $\omega 10 c$ & 38.9 & 6.8 & 8.1 & 7.1 & - \\
\hline $18: 1 \omega 6 c$ and/or $18: 1 \omega 7 c$ & - & 22.1 & 59.3 & 36.1 & - \\
\hline
\end{tabular}

is not produced from glycerol, erythritol, D-arabinose, Larabinose, L-xylose, methyl $\beta$-D-xylopyranoside, sorbose, rhamnose, dulcitol, inositol, methyl $\alpha$-D-mannopyranoside, methyl $\alpha$-D-glucopyranoside, amygdalin, arbutin, lactose, melibiose, inulin, melezitose, raffinose, starch, glycogen, xylitol, turanose, lyxose, tagatose, D-fucose, L-fucose, Darabitol, L-arabitol, gluconate, 2-ketogluconate or 5-ketogluconate. Aesculin is hydrolysed.

The type strain is $3 \cdot 1 \cdot 1^{\mathrm{T}}$ (=LMG $26013^{\mathrm{T}}=\mathrm{NCIMB} 14687^{\mathrm{T}}$ ). The DNA G + C content of strain $3 \cdot 1.1^{\mathrm{T}}$ is $46.6 \mathrm{~mol} \%$.

\section{Acknowledgements}

This work was supported by Program for Changjiang Scholars and Innovative Research Team in University (IRT0959) and Science Foundation of North-East Agricultural University of China and National Natural Science Foundation of China (31101342).

\section{References}

An, D., Cai, S. \& Dong, X. (2006). Actinomyces ruminicola sp. nov., isolated from cattle rumen. Int J Syst Evol Microbiol 56, 2043-2048.

Bringel, F., Castioni, A., Olukoya, D. K., Felis, G. E., Torriani, S. \& Dellaglio, F. (2005). Lactobacillus plantarum subsp. argentoratensis subsp. nov., isolated from vegetable matrices. Int J Syst Evol Microbiol 55, 1629-1634.

Curk, M.-C., Hubert, J.-C. \& Bringel, F. (1996). Lactobacillus paraplantarum sp. nov., a new species related to Lactobacillus plantarum. Int J Syst Bacteriol 46, 595-598. 
De Bruyne, K., Schillinger, U., Caroline, L., Boehringer, B., Cleenwerck, I., Vancanneyt, M., De Vuyst, L., Franz, C. M. A. P. \& Vandamme, P. (2007). Leuconostoc holzapfelii sp. nov., isolated from Ethiopian coffee fermentation and assessment of sequence analysis of housekeeping genes for delineation of Leuconostoc species. Int J Syst Evol Microbiol 57, 2952-2959.

De Bruyne, K., Camu, N., De Vuyst, L. \& Vandamme, P. (2009). Lactobacillus fabifermentans sp. nov. and Lactobacillus cacaonum sp. nov., isolated from Ghanaian cocoa fermentations. Int J Syst Evol Microbiol 59, 7-12.

De Ley, J. (1970). Reexamination of the association between melting point, buoyant density, and chemical base composition of deoxyribonucleic acid. J Bacteriol 101, 738-754.

De Ley, J., Cattoir, H. \& Reynaerts, A. (1970). The quantitative measurement of DNA hybridization from renaturation rates. Eur $J$ Biochem 12, 133-142.

De Man, J. C., Rogosa, M. \& Sharpe, M. E. (1960). A medium for the cultivation of lactobacilli. J Appl Bacteriol 23, 130-135.

Felis, G. E. \& Dellaglio, F. (2007). Taxonomy of Lactobacilli and Bifidobacteria. Curr Issues Intest Microbiol 8, 44-61.
Garrity, G. M., Bell, J. A. \& Lilburn, T. M. (2004). Taxonomic outline of the prokaryotes. In Bergey's Manual of Systematic Bacteriology, 2nd edition, release 5.0. New York: Springer. http://www.bergeys.org/ outlines.html.

Huang, C.-H., Lee, F.-L. \& Liou, J.-S. (2010). Rapid discrimination and classification of the Lactobacillus plantarum group based on a partial dnaK sequence and DNA fingerprinting techniques. Antonie van Leeuwenhoek 97, 289-296.

Naser, S. M., Thompson, F. L., Hoste, B., Gevers, D., Dawyndt, P., Vancanneyt, M. \& Swings, J. (2005). Application of multilocus sequence analysis (MLSA) for rapid identification of Enterococcus species based on rpoA and pheS genes. Microbiology 151, 2141-2150.

Sasser, M. (1990). Identification of Bacteria by Gas Chromatography of Cellular Fatty Acids. Newark, DE: MIDI Inc.

Tamura, K., Dudley, J., Nei, M. \& Kumar, S. (2007). MEGA4: molecular evolutionary genetics analysis (MEGA) software version 4.0. Mol Biol Evol 24, 1596-1599.

Zanoni, P., Farrow, J. A. E., Phillips, B. A. \& Collins, M. D. (1987). Lactobacillus pentosus (Fred, Peterson, and Anderson) sp. nov., nom. rev. Int J Syst Bacteriol 37, 339-341. 